\title{
Much ado about the things we do
}

The Things We Do: Using the Lessons of Bernard and Darwin to Understand the What, How, and Why of Our Behavior by Gary Cziko, A Bradford Book, The MIT Press, Cambridge, 2000. \$37.95 (290 pages) ISBN 0262032775

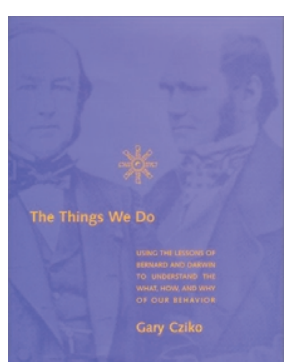

How and why are you reading TINS right now? A Skinnerian view would point out that you might have read this or a similar journal before and that the outcome was rewarding for you, for example your colleagues might have appreciated your broad knowledge base derived from reading TINS articles. Or you might simply enjoy new knowledge, out of curiosity. Incentives increase the frequency of the behavior, punishment decreases it. Instrumental learning is a useful model that allows making predictions about behavior and can explain proximate aspects of it. Although the power of this model might have been overestimated in the past, nobody seriously believes that the traditional divisions to conceptualize learning habituation, instrumental and classical conditioning, and perceptual learning are sufficient to predict and understand behavior. When respective mainstream psychology is criticized by Gary Cziko in his book, he is attacking a straw man. There is no physicist that believes that classical mechanics can provide a sufficient framework to understand the dynamics of matter - although there once might have been - and there is no psychologist who believes that instrumental learning is sufficient to understand the dynamics of behavior although there once might have been. In both cases progress results from more elaborate and broader theories. These must encompass the earlier and more restricted model, and explain the experimental data that have supported the earlier, cruder model. Classical 
mechanics can be derived from quantum theory as a fair approximation under appropriate conditions. No engineer, however, would set up a Schrödinger equation to explain the motion of a clock pendulum. In a similar manner, the pecking behavior of a pigeon in a Skinner box is well approximated by the laws of operant learning. If we want to explain different, more complex conditions, be it the scattering of two electrons or the attempts of Romeo to approach Julia, we have to broaden and change our models.

When the change has reached a certain level, when a new 'species of theories' has arrived, we might call it a revolution or a paradigmatic shift. In a way there is an evolution of theories much like the evolution of species. Cziko is right in suggesting that such a revolution in behavioral sciences is needed, and he suggests the way to proceed. However, like any revoluzzer, he tends to pour the baby with the bathwater. But, don't you have to overstate the point to convince those stubborn conservatives? So let's leave the critique aside that says the author of the book would not sufficiently attempt to base his new avenues on existing roads.

Which way should the evolution of behavioral sciences go? Cziko suggests that the greatest challenge when constructing a new paradigmatic framework is already fulfilled if we take into account three lessons: (1) The Darwinian lesson, meaning to acknowledge the biological information base that all organisms are essentially born with; (2) The Bernardian* lesson suggesting that animate behavior is determined by the purposeful control of perception; and (3) the extended Darwinian lesson, namely that the process of learning involves within-organism selection.

In particular, the Bernardian lesson, which Cziko develops into a cybernetic framework explaining aspects of behavior, is the strength of this book. Cognition is no longer the planning of responses to certain stimuli (ultimate behavior) but rather the reorganization of Bernardian perceptual control systems that control new aspects of the environment. This reorganization and the acquisition of knowledge would then follow Darwinian principles of evolution. Neither a one-way cause-effect (contingency of reinforcer-probability of behavior) logic nor a teleological intention can explain why and how you are still reading this TINS issue, whereas a cybernetic model of a 'purposeful control of your perception' can explain what, how and why of behavior. If we can adequately specify the goal - say the intake of relevant information on the current book marketwe can specify your behavior. As it gets darker during the early evening while you are reading this journal, you might switch on the light or you might use your glasses. If your children have switched on the TV you might leave the living room and go to your study - all of this behavior can be explained by having the one goal in your mind: YOU MUST READ THOSE TINS BOOK REVIEWS! You purposefully control your perception to fulfill this goal; you control your environment so that this purpose can be achieved (Bernardian lesson). And the ways you find to do this follows the extended Darwinian lesson: learning involves within-organism selection. But whether you steal the reading glasses of your partner or whether you go upstairs to search for your own, some aspects of behavior might well be explained by instrumental learning. The Skinnerian view is not, as supposed by Cziko, a cause-effect trap, it is the causa efficiens side of the coin. And already Aristotle has added the other side of the coin, the causa finalis, the telos, as a scientific principle to grasp the intention and purpose of behavior. For both sides of the coin, there is a wealth of data that have been adequately modeled and that must be incorporated in a broader framework. Although not elaborated in the book, the Cziko approach has the potential to achieve this. With the way the author extends Bernard's thinking, elaborating a cybernetic framework for the mind, both sides of the coin, the causa efficiens and the causa finalis are viewed within one model: they become two of many perspectives from which one can look at the dynamic self-regulatory and self-organizing system, called human.

An emphasis on these two projections might have helped to operationalize the new theoretical framework. Insufficient clarity for experimental testing seems a major drawback of Czika's current framework. If not testable, theories can be too good: the theory about the origin of species is one of them, as it can explain everything - think of ways to falsify it! Bringing too much Darwinian lesson into psychology includes the danger to throw us back to the time when we had a way to analyze psycho that could explain everything - post-hoc only, of course.

Much of the book is written in a popular, sometimes simplistic style making it hard for the scholarly reader to extract and accept the essential message that the author wants to transmit. The deep thoughts required by new paradigmatic ideas are often sidetracked when a reader might question some of the more trivial statements in the book. It would be very valuable if Cziko would provide a condensed version for the scientific expert readership, including the derivation of testable experimental hypotheses. Till then you will have to construct your own excerpt from the book - a task that might well be worth doing. In a time where cognitive telos is becoming mainstream fashion, the book by Cziko suggests a refreshing paradigmatic shift: if we want progress in explaining behavior, the marriage of cognitive science with cybernetics will be necessary to broaden limited cognition.

\section{Thomas Elbert}

Center for the Study of Emotion and Attention, University of Florida, Box 100165, Gainesville, FL 32610, USA.

e-mail: Thomas.Elbert@Uni-Konstanz.de

\footnotetext{
*Claude Bernard (1813-1878) recognized that internal processes (Milieu interieur) like body temperature, blood pressure or saline concentration are maintained within certain boundaries in spite of disturbing forces from inside or outside. Gary Cziko argues that living organisms not only control the internal milieu but also aspects of their external environment.
} 